\title{
GammeV and GammeV-CHASE
}

\author{
W. Wester \\ Fermilab, MS209, Batavia IL, USA \\ on behalf of the GammeV and GammeV-CHASE Collaborations
}

\section{Goal of the experiments}

Physics beyond the Standard Model might include Weakly Interacting Slim Particles (WISPs) that address questions such as what is the nature of dark matter or even shed insight into the underlying nature of dark energy. WISPs are a general class of particles that include axions, axion-like particles, hidden sector photons, milli-charged particles, chameleons, etc. The

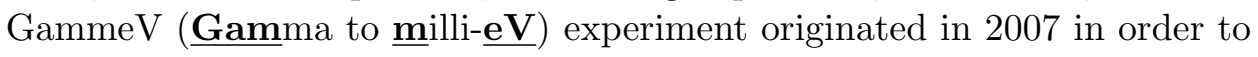
test a positive anomalous axion-like particle interpretation of the PVLAS experiment which was not evident in subsequent data [1]. The experiment was also motivated as it was realized that the milli-eV scale appears naturally in a see-saw between the electroweak and Planck scales, neutrino mass differences, the dark energy density, and the possible mass for certain dark matter candidates. GammeV was first to exclude both a scalar and pseudoscalar axion-like particle interpretation of the anomalous PVLAS result setting a limit of around $3.1 \times 10^{-7} \mathrm{GeV}^{-1}$ on the coupling to photons for low mass axion-like particles.

It has also been found that the parameter space of a variety of other WISP candidates is both largely unexplored and is accessible by modest experiments employing lasers and possibly accelerator magnets. GammeV data has also been used to set limits on possible hidden sector photons [2].

Further work by the GammeV team has focused on a reconfiguration of the apparatus to be sensitive to possible chameleon particles. Chameleons are scalar (or pseudoscalar) particles that couple to the stress energy tensor in a potential such that their properties depend on their environment. In particular, a chameleon acquires an effective mass which increases with local matter density, $\rho$. For a certain class of such potentials, the chameleon field has properties that might explain dark energy [3]. GammeV set the first limits on the coupling of chameleons to photons. A dedicated follow-up

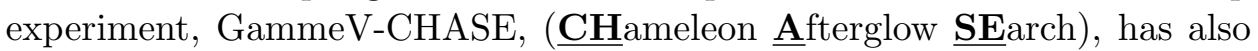
been performed and sets limits on both photon and some model dependent matter couplings as a function of an effective chameleon mass. 


\section{Experimental setup}

The GammeV and GammeV-CHASE apparati are shown in Fig. 1. Both experiments used a $3 \mathrm{~W}$ pulsed Nd:YAG laser frequency doubled to $532 \mathrm{~nm}$ shown into the bore of a Tevatron dipole magnet operating at $5 \mathrm{~T}$. The GammeV experiment used a light shining through a wall (LSW) [4] configuration where a photon propagating in a magnetic field could oscillate into an axion-like particle, traverse an opaque barrier, and have a small probabilty for reconverting back into a detectable photon. Two novel aspects were employed in order to increase sensitivity over the region of interest. A "plunger" was constructed so that the wall could be placed either in the middle or toward one end of the magnet in order to remove regions of insensitivity of mass where the oscillation probability vanishes. The second aspect utilized time correlated single photon counting techniques in order to reject noise from an already relatively low-noise photomultiper tube (PMT). An a priori 10 ns wide search window was established using a calibration run where laser photons were attenuated by approximately 19 orders of magnitude (i.e. no wall) and allowed to be recorded by the PMT.

For GammeV-CHASE, a "particle trapped in a jar" afterglow technique was employed $[5,6]$. The configuration improved many different experimental aspects from the first look by GammeV to extend sensitivity. Laser photons converting into chameleons would remain trapped in the vacuum system until possibly reconverting back into photons that could leave the vacuum system through an optical window and hit the PMT. Similar to the plunger, GammeV-CHASE divided the magnetic field region into three separate regions providing sentivity to different effective chameleon masses. A small residual $\sim 1 \mathrm{~Hz}$ rate of photons from an ion pump and a long-lived luminescence of orange photons similar to known phosphorescence of vacuum grease [7] provided calibration of the experiment's sensitivity.

\section{Accelerator or Lab Facility}

The experiments were conducted at Fermilab's Magnet Test Facility where existing magnet test stands capable of supporting high field operation of Tevatron magnets are located. Besides the magnets and the test facility, the experiments used an existing laser that formally was used by the Accelerator Division and a data acquisition system that Fermilab developed in part for readout of cosmic ray panels as part of the QuarkNet educational program. 

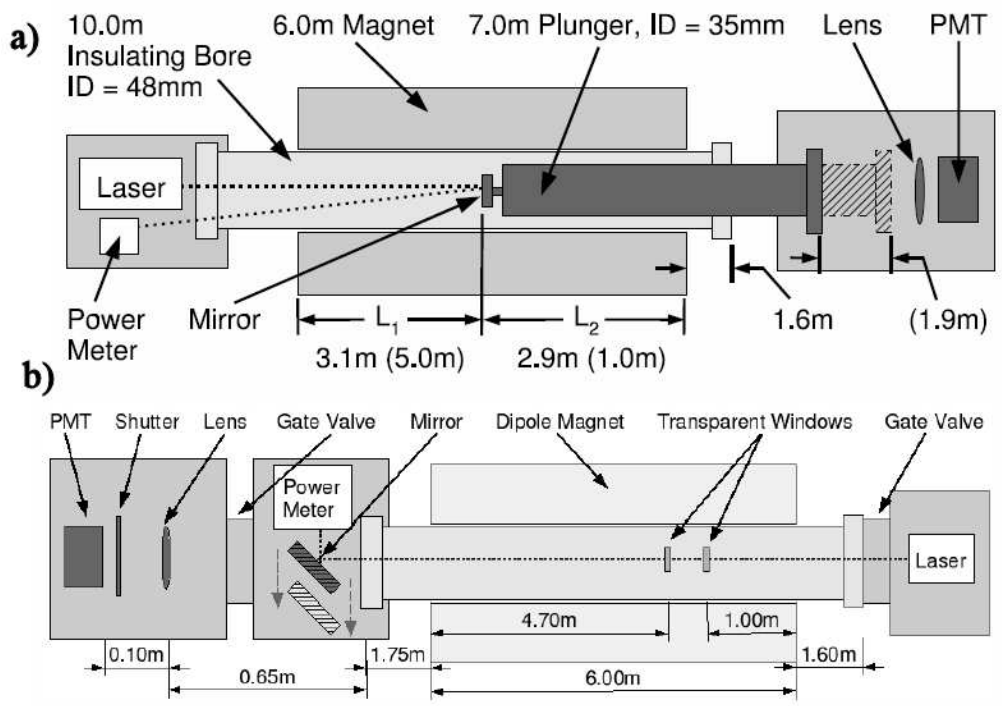

Figure 1: (a) Schematic diagram of the (a) GammeV experimental apparatus and (b) GammeV-CHASE experimental apparatus. The laser, spare Tevatron magnet, phototubes, and data acquition electronics were used by both experiments.

\section{Physics Reach}

Both GammeV and GammeV-CHASE observed no significant WISP signal above background. Fig. 2 shows the exclusion regions of the coupling to photons versus the (effective) mass for axion-like particles $(3 \sigma)$ and chameleons (95\% C.L.). For GammeV, data was recorded in two polarizations for two configurations of the position of the wall with each of these runs consisting of approximately 20 hours of magnet time. The non-observation of a signal has also been reported by other experiments worldwide [9]. For GammeVCHASE, multiple runs were recorded with 10 hours to reach the smallest couplings of photons to chameleons. Shorter runs were recorded at reduced magnetic field to cover larger couplings of photons to chameleons.

The reach of the GammeV and GammeV-CHASE essentially was an energy scale of a $3 \times 1000 \mathrm{TeV}$ and $3 \times 10000 \mathrm{TeV}$ respectively. These high scales are made accessible by the intense photon beam (incident photons were a few $\times 10^{23}$ ) from which a small regenerated photon signal could be identified above background. 

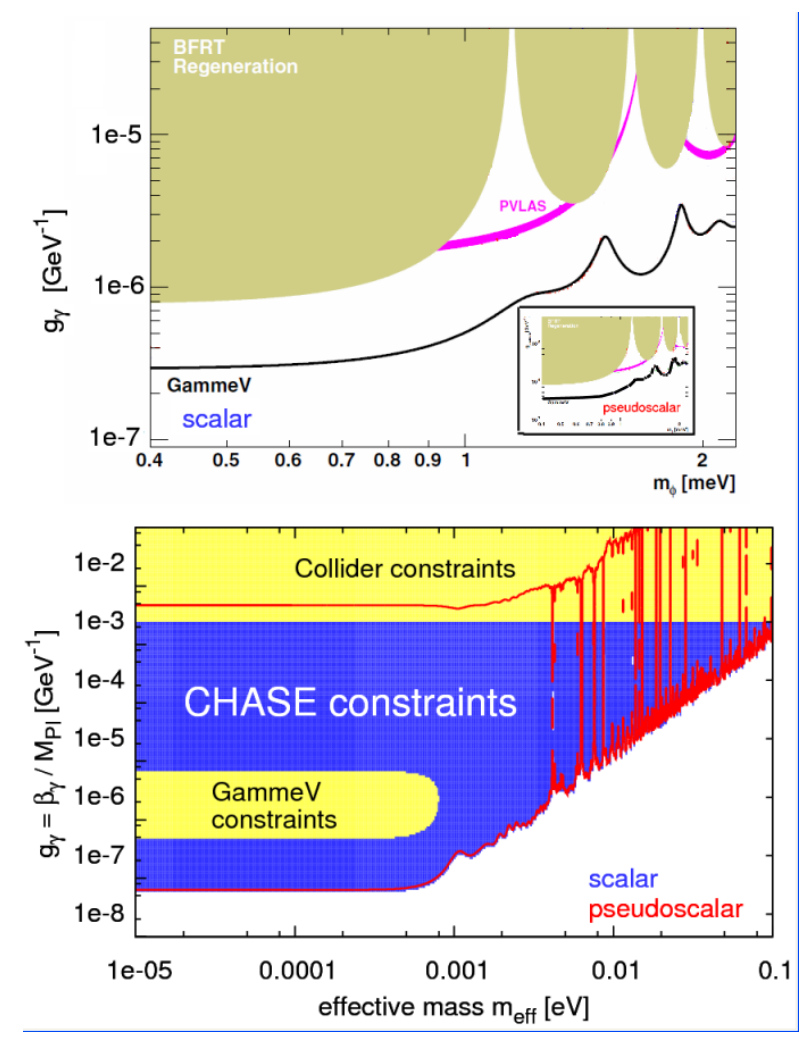

Figure 2: Published exclusion regions of photon coupling versus effective mass obtained (a) by GammeV for scalar and pseudoscalar (inset) axion-like particles at $3 \sigma$ and (b) by GammeV and GammeV-CHASE for chameleon particles at $95 \%$ C.L. where the GammeV and GammeV-CHASE regions are valid for $\eta>0.8$ and 0.1 respectively when expressing $m_{e f f}>\rho^{\eta}$.

\section{$5 \quad$ Status and Schedule}

The experiments are completed and the results have been published. The GammeV goal has been achieved of excluding the WISP interpretation of the PVLAS anomolous signal [8] and setting first limits on chameleon photon coupling [5]. The GammeV-CHASE experiment achieved its goal [10] of extending the region of exclusion for chameleon photon couplings valid for a larger range of potential models [11]. 


\section{$6 \quad$ Future Plans}

The future plans include extending the GammeV region of sensitivity from a few $\times 10^{-7} \mathrm{GeV}^{-1}$ to possibly the $10^{-12} \mathrm{GeV}^{-1}$ level. Such an effort has been called REAPR [12], Resonnantly Enhanced $\underline{\text { Axion-Photon }}$ Regeneration." This possible project is described elsewhere in this document. There is on-going R\&D on long baseline optical cavities in conjuction with the Holometer [13] experiment.

There are ideas of extending the search region for chameleons as well as hidden sector photons. Before an experiment is proposed, careful consideration will be given to the increase in sensitivity compared with other experimental results and the motivation for and effort that it would take to obtain that sensitivity.

\section{Collaborating Institutions and Collaborators}

A. S. Chou ${ }^{1,2}$, W. Wester ${ }^{1}$, A. Baumbaugh ${ }^{1}$, H. R. Gustafson ${ }^{3}$, Y. Irizarry-Valle ${ }^{1}$, P. O. Mazur ${ }^{1}$, J. H. Steffen ${ }^{1}$, R. Tomlin ${ }^{1}$, X. Yang ${ }^{1}$, J. Yoo ${ }^{1}$ (GammeV Collaboration)

${ }^{1}$ Fermi National Accelerator Laboratory, PO Box 500, Batavia, IL 60510,

${ }^{2}$ Center for Cosmology and Particle Physics, New York University, 4 Washington Place, New York, NY 10003, ${ }^{3}$ Department of Physics, University of Michigan, 450 Church St, Ann Arbor, MI 48109

J. H. Steffen ${ }^{1}$, A. Upadhye ${ }^{2}$, A. Baumbaugh ${ }^{1}$, A. S. Chou ${ }^{1}$, P. O. Mazur ${ }^{1}$, R. Tomlin ${ }^{1}$, A. Weltman ${ }^{3}$, and W. Wester ${ }^{1}$

(GammeV-CHASE Collaboration)

${ }^{1}$ Fermi National Accelerator Laboratory, PO Box 500, Batavia, IL 60510,

${ }^{2}$ Argonne National Laboratory, 9700 South Cass Ave., Lemont, IL 60437,

${ }^{3}$ Astrophysics, Cosmology and Gravity Centre, Univ. of Cape Town, Rondebosch, Private Bag, 7700, South Africa

\section{References}

[1] E. Zavattini et al. [PVLAS Collaboration], Phys. Rev. Lett. 96, 110406 (2006) [arXiv:hep-ex/0507107]. E. Zavattini et al. [PVLAS Collaboration], E. Zavattini et al. [PVLAS Collaboration], Phys. Rev. D 77, 032006 (2008) [arXiv:0706.3419 [hep-ex]]. 
[2] M. Ahlers, H. Gies, J. Jaeckel, J. Redondo and A. Ringwald, Phys. Rev. D 77, 095001 (2008) [arXiv:0711.4991 [hep-ph]].

[3] J. Khoury, A. Weltman, Phys. Rev. D69, 044026 (2004). [astro$\mathrm{ph} / 0309411]$.

[4] K. Van Bibber, N. R. Dagdeviren, S. E. Koonin, A. K. Kerman and H. N. Nelson, Phys. Rev. Lett. 59, 759 (1987).

[5] A. S. Chou et al. [ GammeV Collaboration ], Phys. Rev. Lett. 102, 030402 (2009). [arXiv:0806.2438 [hep-ex]].

[6] M. Ahlers, A. Lindner, A. Ringwald, L. Schrempp, C. Weniger, Phys. Rev. D77, 015018 (2008). [arXiv:0710.1555 [hep-ph]].

[7] D.W. Cooke and B.L. Bennett, "Long-lived luminescence from commonly used Apiezon compounds", Journal of Luminescence, Volume 65,283 (1995).

[8] A.S. Chou et al. [GammeV Collaboration], Phys. Rev. Lett. 100, 080402 (2008). Additional description may be found at: http://gammev.fnal.gov.

[9] M. Fouche et al. [BMV Collaboration], Phys. Rev. D 78, 032013 (2008) [arXiv:0808.2800 [hep-ex]].

K. Ehret et al. [ALPS Collaboration], Phys. Lett. B 689, 149 (2010).

A. Afanasev et al. [LIPSS Collaboration], Phys. Rev. Lett. 101, 120401 (2008) [arXiv:0806.2631 [hep-ex]].

P. Pugnat et al. [OSQAR Collaboration], Phys. Rev. D 78, 092003 (2008) [arXiv:0712.3362 [hep-ex]].

[10] A. Upadhye, J. H. Steffen and A. Weltman, Phys. Rev. D 81, 015013 (2010) [arXiv:0911.3906 [hep-ph]].

[11] J. H. Steffen et al., Phys. Rev. Lett. 105, 261803 (2010) [arXiv:1010.0988 [astro-ph.CO]].

[12] G. Mueller, P. Sikivie, D. B. Tanner and K. van Bibber, Phys. Rev. D 80, 072004 (2009) [arXiv:0907.5387 [hep-ph]].

[13] C. J. Hogan, arXiv:0905.4803 [gr-qc]. See also: www.fnal.gov/directorate/program_planning/Nov2009PACPublic/holometerproposal-2009.pdf. 